\title{
Substrate proteolysis is inhibited by dominant- negative Nedd4 and Rsp5 mutants harboring alterations in WW domain 1
}

\author{
Natalia Shcherbik ${ }^{1}$, Sharad Kumar ${ }^{2}$ and Dale S. Haines ${ }^{1 *}$ \\ ${ }^{1}$ Fels Institute for Cancer Research and Molecular Biology, Temple University School of Medicine, 3307 N. Broad Street, Philadelphia, PA 19140 , \\ USA \\ ${ }^{2}$ Hanson Centre for Cancer Research, Institute of Medical and Veterinary Science, PO Box 14, Rundle Mall, Adelaide SA 5000, Australia \\ Author for correspondence (e-mail: dhaines@unix.temple.edu)
}

Accepted 28 November 2001

Journal of Cell Science 115, 1041-1048 (2002) @ The Company of Biologists Ltd

\section{Summary}

Mammalian Nedd4 and its budding yeast orthologue Rsp5 are members of a large family of HECT-domain-containing ubiquitin ligases. Besides possessing a $\mathrm{Ca}^{2+} /$ lipid-binding domain, both ligases have multiple protein-interacting modules termed WW domains. The C-terminal WW domains mediate interactions with substrates, but the function of the first $W W$ domain remains unclear. We found that expression of a WW domain 1 Nedd4 mutant inhibits the growth of budding yeast by affecting the rsp5ole1 pathway. The WW domain 1 mutant-induced phenotype is suppressed by ole1 cDNA overexpression or oleic acid supplementation of growth media and ole1 RNA levels are reduced in cells expressing this Nedd4 mutant. Also, the WW domain 1 Nedd4 mutant associates via WW domains 2 and 3 with Spt23, a Rsp5 target and ole1 transactivator. The dominant-negative activity of this mutant is associated with promoting accumulation of unprocessed Spt23 and inhibiting generation of processed and presumably active protein. Also, Spt23 processing is inhibited by a Nedd4 mutant that lacks ubiquitin ligase activity and Spt23-binding-competent Rsp5 mutants harboring WW domain 1 or ligase domain mutations. Interestingly, in mammalian cells, wild-type Nedd4 promotes proteasome-mediated degradation of the precursor form of Spt23. WW domain 1 and ligase domain Nedd4 mutants block its degradation. These results indicate that WW domain 1 of these ligases interacts with cofactors that are required for ubiquitin/proteasomedependent proteolysis of bound substrates.

Key words: Nedd4, Rsp5, WW domain, ole1, Spt23

\section{Introduction}

Ubiquitination is a modification process that regulates the abundance and activity of a large percentage of eukaryotic proteins. Ubiquitination targets proteins for degradation by the proteasome or the lysosome (Hershko and Ciechanover, 1998; Hicke, 1999; Pickart, 2001). Interestingly, recent studies have shown that ubiquitination regulates the activity of proteins by mechanisms that are independent of complete degradation. For example, the budding yeast Met4 transcription factor is inactivated, but not degraded, upon ubiquitination (Kaiser et al., 2000), whereas the kinase activity of mammalian TAK1 is induced by ubiquitination (Wang et al., 2001). Other examples include ubiquitination promoting limited proteasomedependent processing of membrane-bound transcription factors Spt23 and Mga2 in budding yeast and the NF- $\mathrm{KB}$ precursor p105 in mammalian cells (Palombella et al.,1994; Hoppe et al., 2000). Processed proteins either translocate to the nucleus where they function as transactivators (as in the case of Spt23 and $\mathrm{Mga} 2$ ) or are sequestered in a latent state by an inhibitor in the cytosol (as in the case of p105).

Covalent attachment of ubiquitin to a protein substrate requires the coordinated activity of numerous proteins. These include a ubiquitin-activating enzyme (E1), a ubiquitinconjugating enzyme (E2) and a specificity factor that functions in substrate binding (an E3 or ubiquitin ligase) (Hershko and Ciechanover, 1998; Pickart, 2001). One major subclass of ubiquitin ligases is the HECT (for Homologous to E6-AP CTerminus)-domain-containing proteins (Huibregtse et al., 1995). Unlike other types of ubiquitin ligases, substrate ubiquitination by HECT-domain-containing enzymes requires the intermolecular transfer of ubiquitin from the bound E2 to the E3 prior to attachment onto the target. This transfer is dependent on the formation of a thioester bond between ubiquitin and a conserved Cys residue that is localized at the C-terminus of the HECT domain (Hershko and Ciechanover, 1998; Pickart, 2001).

A number of HECT domain ubiquitin ligases have been identified that possess multiple WW domains and a $\mathrm{Ca}^{2+} /$ lipidbinding (C2) domain (Harvey and Kumar, 1999). These proteins, termed the Nedd4 family, have been cloned from yeast, Caenorhabditis elegans, Xenopus leavis, rodents and man and include Rsp5, Pub1, Itch, AIP-4, WWP1/AIP5, WWP2/AIP2, Nedd4, Smurf-1, Smurf-2, KIA0322 and KIAA0439. The WW domain is a protein-interacting module, and it binds to at least four different types of sequence (Sudol and Hunter, 2000). These are polypeptides with core amino acids Pro-Pro-X-Tyr or Pro-Pro-Leu-Pro, polyproline residues that are flanked by Arg or Lys, and peptides that are 
phosphorylated on Ser or Thr residues. A C2 domain mediates translocation of proteins to phospholipid membranes in response to increased cytosolic $\mathrm{Ca}^{2+}$ (Rizo and Südhof, 1998).

Orthologous Rsp5 and Nedd4 are the best studied of the Nedd4 family. Although only a limited number of Nedd4 substrates have been identified (Staub et al., 1996; Abriel et al., 2000; Hamilton et al., 2001; Pham and Rotin, 2001), Rsp5 has been implicated in the ubiquitination and degradation of many proteins (Huibregtse et al., 1997; Erdeniz et al., 2000; Rotin et al., 2000; Andoh and et al., 2000). Recently, the essential function of Rsp5 has been linked to ole 1 gene transactivation (Hoppe et al., 2000). Rsp5 induces 'regulated ubiquitin/ proteasomal-dependent processing' (termed RUP) of Spt23, one of two ER-membrane-bound proteins that are positive regulators of ole1 gene expression (Zhang et al., 1999; Hoppe et al., 2000). Processed Spt 23 is released from the ER membrane and translocates to the nucleus, where it enhances olel gene transcription (Hoppe et al., 2000; Hitchcock et al., 2001). Although it is widely accepted that the C2 domain promotes membrane localization of Rsp5 and Nedd4 (Plant et al., 1997; Plant et al., 2000; Wang et al., 2001) and their C-terminal WW domains mediate binding to substrates (Staub et al., 1996; Harvey et al., 1999; Chang et al., 2000; Farr et al., 2000; Hoppe et al., 2000), the function of the highly conserved WW domain 1 remains undefined.

Results presented here indicate that WW domain 1 binds cofactors involved in ubiquitin/proteasome-dependent proteolysis of substrates. We have found that Spt23 processing is inhibited in budding yeast by Nedd4 and Rsp5 mutants harboring alterations in WW domain 1 or the ligase domain. In transfected mammalian cells, wild-type (wt) Nedd4 promotes proteasome-mediated degradation of precursor Spt23 whereas WW-domain1- and ligase-defective Nedd4 mutants block degradation. Because we are able to easily detect an interaction between the WW domain 1 Nedd4/Rsp5 mutants and Spt23 in cells, it is likely that disruption of WW-domain1binding function eliminates E3-induced degradation but not substrate-binding activity. These mutational studies point to the existence of Nedd4- and Rsp5 WW-domain1-binding proteins that are required for proteasome-dependent degradation and/or processing of substrates.

\section{Materials and Methods}

\section{Yeast strain and yeast expression constructs}

The Saccharomyces cerevisiae InvSc1 strain (Mat-a his3 $\Delta 1$ leu2 trp1289 ura3-52) was purchased from Invitrogen. Full-length Nedd4 was cloned by RT-PCR using RNA prepared from normal human lymphocytes and primers (sequences of all primers employed in this study are available upon request) spanning the coding region of Nedd4. Site-directed mutagenesis procedure was performed to generate the various Nedd4 WW domain and HECT domain mutants using pYes (Invitrogen, contains a galactose-inducible promoter and the $u$ ra 3 selectable marker)-wildtype (wt) Nedd4 as the template. PCR reactions were typically carried out in a $50 \mu \mathrm{l}$ reaction containing $1 \times$ PFU buffer (Stratagene), pYes-wt Nedd4, 20 pmol primers that contain appropriate mis-matches to the wild-type sequence, $0.8 \mathrm{mM}$ dNTPs and 2 units of the low error rate thermostable polymerase PFU (Stratagene) for the following cycles: 18 cycles at $94^{\circ} \mathrm{C}$ for 30 seconds, $55^{\circ} \mathrm{C}$ for 60 seconds and $72^{\circ} \mathrm{C}$ for 16 minutes. After PCR reactions were completed, 10 units of the DpnI restriction endonuclease were added to each reaction and samples were placed at $37^{\circ} \mathrm{C}$ for 1 hour. DNA was transformed into ultracompetent XL-1
E. coli (Stratagene). Plasmid DNA was prepared from individual recombinants and sequenced to identify those harboring the desired mutation. At least two sequenced-verified mutants were tested for their activity in yeast. To generate the Nedd 4 WW domain 1 deletion mutant, PFU-based PCR was used to amplify sequences immediately upstream and downstream of WW domain 1 using pYes-wt Nedd4 as the template. A BamHI site was introduced into the $3^{\prime}$ primer of the $\mathrm{N}$-terminal set and into the $5^{\prime}$ primer of the C-terminal set. The $\mathrm{N}$ terminal portion was cloned into pYes, followed by cloning of the Cterminal portion in-frame into pYes harboring the $\mathrm{N}$-terminal fragment. Again, at least two different sequenced verified mutants were tested for their activity in yeast. The pESC-LEU (Stratagene, contains a galactose-inducible promoter and the leu2 selectable marker) Nedd 4 constructs were generated via subcloning of the Nedd4 inserts from pYes.

The FLAG-tagged Spt 23 construct was generated by cloning PCR product (generated with primers that span the coding region of the gene, genomic DNA prepared from the InvSc1 strain and PFU) inframe with the FLAG tag present (N-terminal to Spt23) in the pESCLEU2 vector. The Rsp5 constructs were generated in a similar manner in pYes-BBV-HA using oligodeoxynucleotides amplifying full length Rsp5 or truncated Rsp5 starting immediately C-terminal to WW domain 1 . The $\mathrm{C}$-terminal pYes-Rsp5 mutant contains a stop codon upstream of the $\mathrm{C}$ - terminal Cys residue.

\section{Yeast techniques}

Standard protocols were followed for preparation of yeast media and yeast manipulations (Guthrie and Fink, 1991). For viability assays, yeast were transformed with plasmids by the lithium acetate method and plated onto glucose agar (all media used in described assays included the appropriate drop-out supplements that allows for the selection of transformed yeast with the indicated plasmids). Cells were incubated at $30^{\circ} \mathrm{C}$ for 3 days. Colonies were picked and cells were grown overnight to equal densities in glucose media. Cells were diluted to varying degrees, streaked onto glucose or galactose agar and incubated at $30^{\circ} \mathrm{C}$. To determine the effects of oleic acid on Nedd4-induced toxicity, cells were processed as described above and streaked onto galactose agar supplemented with $0.5 \mathrm{mM}$ oleic acid (made up in $1 \%$ tergitol (NP-35)). For the high copy suppressor screen, pESC-LEU containing the Nedd4 WW domain 1 mutant was transformed into the InvSc1 strain. Cells harboring this construct were subsequently transformed with a yeast expression cDNA library (ATCC; library number 87276) and plated onto galactose agar. Over 400,000 individual yeast cDNAs clones were screened and approximately 100 visible colonies appeared on these plates after 3 days at $30^{\circ} \mathrm{C}$. DNA was isolated from yeast clones retaining Nedd4 expression protein and transformed into ultracompetent XL-1 cells. Multiple colonies were picked from each transformation and grown overnight in ampicillin-containing liquid broth media. Plasmid DNA was prepared and those derived from the yeast cDNA library were identified by a $X h o I$ restriction endonuclease digestion. One clone was chosen from each of the transformants and tested for its ability to reverse the growth-inhibitory activity of the WW domain 1 mutant. Only 7 clones generated a suppressible phenotype. These clones were sequenced and database searches revealed that all clones were derived from the ole1 gene.

\section{Western blots, coimmunoprecipitations and northern blots}

Cells were grown under the appropriate conditions (described in detail in the legend of the Figures), pelleted and stored at $-80^{\circ} \mathrm{C}$ prior to lysis. Cells were lysed in RIPA buffer (50 mM Tris ( $\mathrm{pH} 8.0), 150 \mathrm{mM}$ $\mathrm{NaCl}, 0.1 \%$ SDS, $0.5 \%$ deoxycholic acid and $1 \%$ Nonidet P-40) supplemented with $1 \mathrm{mM}$ PMSF, $2 \mu \mathrm{g} / \mathrm{ml}$ aprotinin, $2 \mu \mathrm{g} / \mathrm{ml}$ leupeptin, $1 \mu \mathrm{g} / \mathrm{ml}$ soy trypsin inhibitor and $1 \mu \mathrm{g} / \mathrm{ml}$ pepstatin A. Acid-washed glass beads (425-600 microns) (Sigma) were added to the lysate to give a $50 \%$ beads/lysate (v/v) mixture. Samples were 
vortexed at $4^{\circ} \mathrm{C}$ for 20 minutes. Lysates were clarified by centrifugation, the supernatant transferred to a new tube and the protein concentration determined by the Bradford method (BioRad). Samples were mixed with $4 \times$ SDS-PAGE sample loading buffer and boiled for 5 minutes. Approximately $10 \mu \mathrm{g}$ of total protein was separated on SDS-polyacrylamide gels and transferred onto nitrocellulose membranes. Blots were probed with rabbit polyclonal antibodies raised against mouse Nedd 4 or commercially available mouse monoclonal antibodies against HA (12CA5, Roche Molecular Biochemicals) or FLAG (M5, Sigma) epitope tags. Signals were visualized using horseradish-peroxidase-conjugated secondary antibodies and enhanced chemiluminescence (NEN). For coimmunoprecipitation assays, cells were grown under the appropriate conditions, pelleted and lysed in RIPA buffer as previously described. After clarification, protein concentration was measured by the Bradford assay and $600 \mu \mathrm{g}$ of protein was pre-cleared for 1 hour with protein-G sepharose (Amersham Pharmacia) at $4^{\circ} \mathrm{C}$. After a brief centrifugation, the supernatant was transferred to a new tube containing $1 \mu \mathrm{g}$ of the immunoprecipitating antibody. After a 4 hour incubation at $4^{\circ} \mathrm{C}$, protein- $\mathrm{G}$ sepharose was added and the mixtures were incubated for an additional 2 hours at $4^{\circ} \mathrm{C}$. Protein complexes were washed three times with cold lysis buffer and resuspended in $1 \times$ SDS-PAGE loading buffer. Samples were placed in a boiling water bath for 5 minutes to elute protein from beads and western blots were performed as described above.

For ole1 transcript analysis, single colonies transformed with a control or Nedd4 expression constructs were picked and grown in glucose media for approximately 36 hours at $30^{\circ} \mathrm{C}$. Cells were pelleted and resuspended in $0.5 \mathrm{mM}$ oleic acid supplemented glucose media and incubated overnight. Cells were again pelleted and resuspended in oleic acid supplemented galactose media. Cells were incubated for an additional 4 hours, pelleted and resuspended in non-supplemented galactose media. Cells were grown for 2 more hours before harvesting and isolation of RNA. RNA was separated on agarose gels containing formaldehyde and transferred to nylon membranes. Hybridizations were performed in Rapid-Hyb (Amersham-Pharmacia) using a ${ }^{32} \mathrm{P}$ radiolabeled ole 1 cDNA probe generated from plasmid DNA obtained via the high copy suppressor screen.

\section{Spt23 expression analysis in mammalian cells}

Nedd 4 and Spt 23 cDNAs were cloned from appropriate yeast expression vectors into the pCEP mammalian expression vector (Invitrogen). $5 \mu \mathrm{g}$ of each construct were transfected in duplicate in the human lung adenocarcinoma H1299 cell line by Lipofectamine (Invitrogen) according to manufacturer's instructions. One set of transfections was washed and re-fed with complete media while the other was re-fed with complete media containing $10 \mu \mathrm{M}$ of the proteasome inhibitor MG115 (Calbiochem). Cells were harvested 9 hours later, and the extract was prepared in RIPA buffer.

\section{Results}

\section{Nedd4 proteins harboring WW domain 1 mutations} suppress the growth of budding yeast

A fragment of human Nedd4 was identified in a yeast twohybrid screen that we performed (Boyd et al., 2000). While investigating an interaction between full-length Nedd4 (generated by RT-PCR) and the bait, we noticed that one of the Nedd4 clones displayed dramatic toxicity in yeast. Sequencing of the clone revealed that it contained multiple mutations. Only one of the changes was present in a known functional domain of Nedd4. Fig. 1 depicts a schematic representation of Nedd4 and the mutation (underlined) present in WW domain 1 of the toxic clone. Interestingly, the mutated Tyr residue within WW
A.

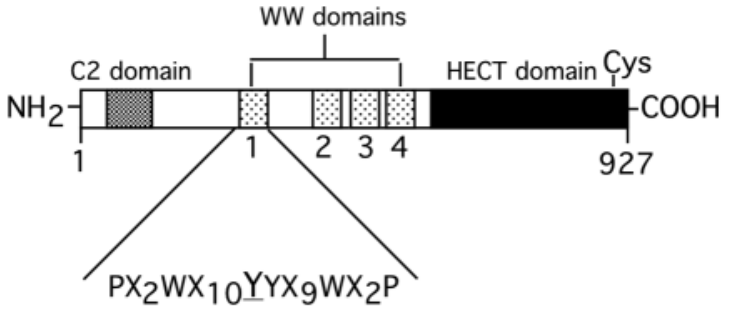

B.

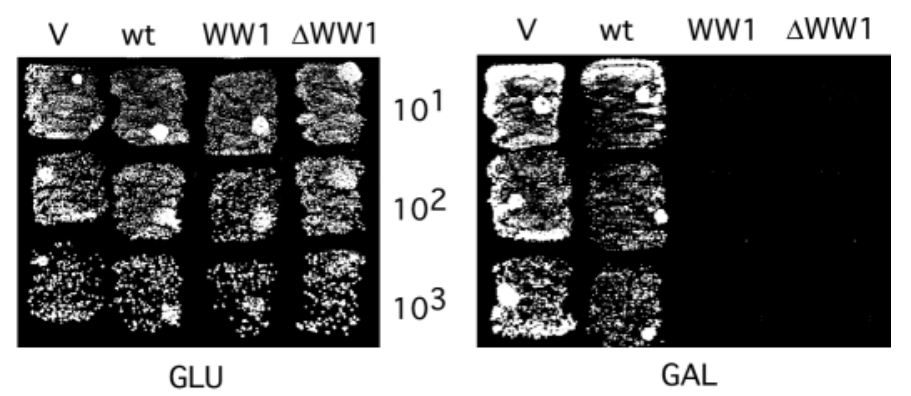

C.

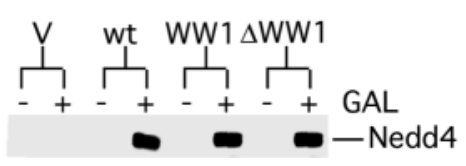

Fig. 1. Nedd4 WW domain 1 mutants block the growth of budding yeast. (A) The known functional domains of human Nedd4 and consensus amino acids of the WW domain are depicted. The amino acid underlined is the residue of WW domain 1 that was mutated in the toxic Nedd4 clone as well as the one that was changed to an Ala by site-directed mutagenesis. (B) Cells were transformed with pYes (V), pYes-wt Nedd4 (wt) and the pYes constructs encoding the WW domain 1 point (WW1) or deletion ( $\Delta \mathrm{WW} 1)$ mutants. Single colonies were picked from plates and grown overnight to equal densities in glucose media. Cells were diluted to varying degrees and streaked onto glucose and galactose agar plates. Cells were incubated for 3 days. It should be noted that multiple clones from each transformation were analyzed and the results obtained were identical to those presented. (C) Cells transformed with the same constructs presented in Fig. 1B were grown in glucose media overnight. Cells were pelleted and resuspended in glucose $(-)$ or galactose $(+)$ media. Cultures were incubated for 8 hours. Cells were pelleted, protein extract was prepared and the amount of Nedd4 was measured by a western blot. Ponceau S staining of blots after transfer revealed equivalent loading of total protein.

domain 1 is highly conserved between WW domains and is critical for protein binding (Lu et al., 1999). To determine if mutation of this residue is responsible for the observed toxicity, we substituted the Tyr with Ala by site-directed mutagenesis using a wild-type (wt) Nedd4 cDNA clone (present in a galactose-inducible expression vector) as the template. Yeast were transformed with wt Nedd4, the WW domain 1 mutant and an empty vector control. After transformation, individual clones were picked, grown in glucose media and streaked at varying dilutions onto glucose and galactose agar plates. As depicted in Fig. 1B, all transformants grew at a similar rate under conditions where Nedd4 was not expressed (i.e. when grown on glucose media). In contrast, cells containing the WW domain 1 mutant were unable to proliferate on galactose media. 
A.

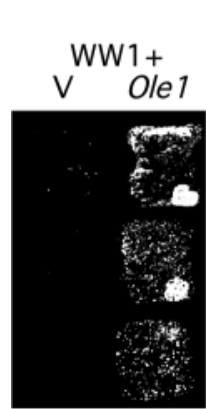

GAL

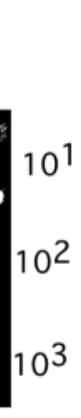

$10^{1}$
$10^{2}$
$0^{3}$

GA
B.

WW1 oleic acid
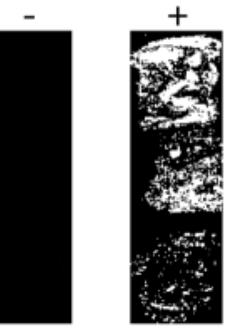

GAL
C.

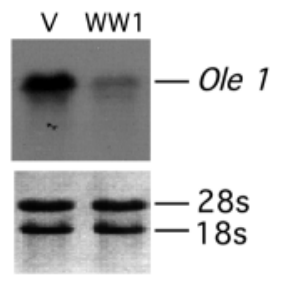

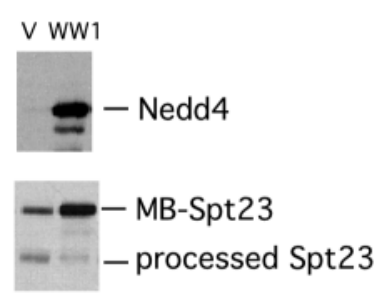

Fig. 2. An olel cDNA or oleic acid suppresses the toxic activity of the WW domain 1 mutant, and the WW domain 1 mutant inhibits ole 1 gene expression and Spt23 processing. (A) Cells containing pYes-WW1 were transformed with the ole1 cDNA expression construct (ole1) or an empty vector control (V). Cells were processed as described in the legend of Fig. 1B. For the oleic acid experiment, cells containing pYesWW1 were plated onto either plain galactose media (-) or galactose media that had been supplemented

with $0.5 \mathrm{mM}$ of this unsaturated fatty acid. (B) Cells transformed with pYes (V) or pYes-WW1 (WW1) were picked and processed as described in the Materials and Methods. RNA was isolated from cells, separated on agarose gels containing formaldehyde, transferred to nylon membrane and an ole1 northern blot was performed. Also depicted is a picture of the ethidium-bromide-stained gel prior to transfer showing equivalent loading of RNA. (C) Cells were transformed first with the pESC-FLAG-Spt23 expression construct and then with pYes (v) or pYes-WW1 (WW1). Transformed cells were grown in glucose media overnight. Cells were pelleted and resuspended in galactose media. Cultures were harvested 6 hours later for the preparation of protein extract. Measurement of Nedd4 protein in the extract was performed by a western blot using the anti-Nedd4 polyclonal antibody while the amount of membrane-bound (MB) and processed Spt23 was measured by the anti-FLAG antibody M5. Ponceau S staining of the blot after transfer revealed equivalent loading of total protein.

Yeast expressing wt Nedd4 grew at a slightly slower rate under conditions used to induce Nedd4 expression when compared with vector-alone-transformed cells.

Although the mutation introduced within WW domain 1 should eliminate its binding function (Lu et al., 1999), it is possible that the toxic activity of the WW domain 1 mutant is dependent on de novo protein association mediated by the mutated WW domain. Therefore, we wanted to determine if a WW domain 1 deletion mutant suppresses the growth of yeast. Fig. 1B demonstrates that Nedd4 with a deleted WW domain 1 inhibits proliferation. Fig. 1C shows a result of a western blot depicting equivalent level of expression of wt Nedd4 and the WW domain 1 mutants after galactose induction. These results indicate that disruption of WW-domain1-binding function induces a toxic activity of human Nedd 4 in budding yeast, and this is not associated with differential expression of the mutant protein.

The WW domain 1 mutant inhibits ole 1 gene expression and Spt23 processing

To investigate a mechanism of WW domain 1 mutant action, we performed a high copy suppressor screen to identify yeast cDNAs that block its toxic function. Of over 400,000 yeast cDNAs screened, only seven suppressors were identified. Sequence analysis of the clones revealed that all were derived from the ole 1 gene. This gene encodes $\Delta 9$ fatty acid desaturase, an essential yeast enzyme involved in the synthesis of oleic acid (Stukey et al., 1989). To test if oleic acid also suppresses the growth inhibitory activity of this mutant, WW-domain1transformed cells were plated onto oleic acid supplemented media. As shown in Fig. 2A, overexpression of an olel cDNA or oleic acid supplementation of growth media blocks the toxic activity of the WW domain 1 mutant.

Rsp5's essential function has recently been linked to $\Delta 9$ fatty acid desaturase production (Hoppe et al., 2000). It has been documented that $r s p 5$-null cells proliferate on oleic acid media and that Rsp5 is required for ole1 RNA synthesis when cells are grown in oleic-acid-free media (Hoppe et al., 2000). These findings prompted us to determine if the Nedd4 WW domain 1 mutant inhibits ole1 RNA production. Yeast transformed with empty vector or the WW domain 1 mutant were grown in oleic-acid-containing media to repress ole 1 transcription. Then, cells were cultured in galactose media lacking oleic acid to induce ole 1 transcription and harvested 2 hours later for isolation of RNA. As shown in Fig. 2B, lower amounts of ole1 transcripts were evident in cells that express the Nedd4 WW domain 1 mutant when compared to yeast transformed with the vector control. These results show that the Nedd4 WW domain 1 mutant inhibits expression of the essential yeast gene ole1.

Rsp5 influences ole1 gene expression by an interesting mechanism. Rsp5 induces ubiquitin/proteasome-dependent processing of Spt23, an ER-bound transcription factor that is a positive regulator of ole1 gene transcription (Zhang et al., 1999; Hoppe et al., 2000; Hitchcock, 2001). Processed Spt23 is released from the ER membrane and translocates to the nucleus, where it presumably functions as an ole 1 gene transactivator. Given that the Nedd4 WW domain 1 mutant is an inhibitor of ole1 gene expression, we next wanted to determine if it alters ubiquitin/proteasome-dependent processing of Spt23. We measured the levels of membrane-bound (larger molecular weight product) and processed (smaller molecular weight product) Spt 23 in cells expressing the WW domain 1 mutant by immunoblotting. Interestingly, cells expressing the WW domain 1 mutant have a higher amount of unprocessed Spt23 and a lower level of processed Spt23 protein (Fig. 2C) when compared to vector-alone-transformed cells. These results show that the Nedd4 WW domain 1 mutant inhibits ubiquitin/proteasomedependent processing of Spt23. Inhibition of processing probably results in lower amounts of transcriptionally active Spt23 protein and reduced levels of olel RNA.

\section{The Nedd4 WW domain 1 mutant interacts with Spt23} via WW domains 2 and 3

As the WW domain 1 mutant promotes accumulation of membrane-bound SPT23, the results above indicate that the 
A.

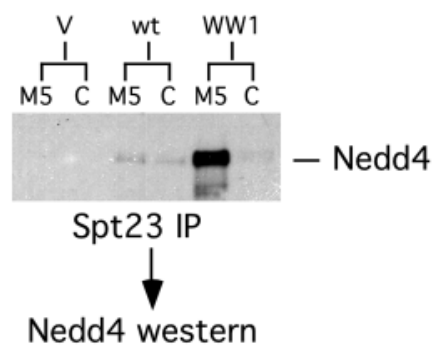

B.

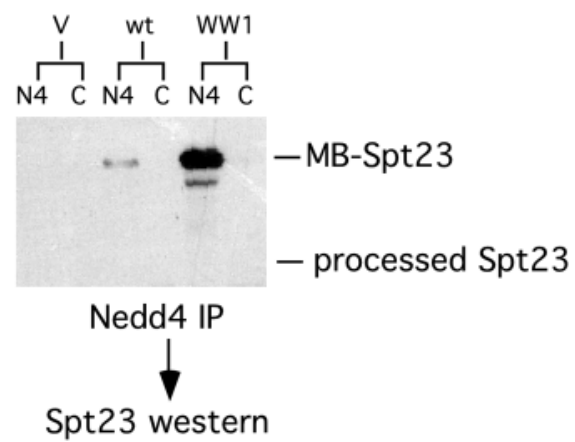

Fig. 3. The WW domain 1 Nedd 4 mutant interacts with membrane-bound Spt23. (A) Cells transformed with Spt23 and the indicated Nedd4 expression constructs or vector alone control (V) were grown as described in the legend of Fig. 2C and lysed in RIPA buffer. The extract ( $600 \mu \mathrm{g}$ per immunoprecipitation reaction) was pre-cleared for 1 hour with protein-G sepharose. After a brief centrifugation, the supernatant was transferred to a new tube containing $1 \mu \mathrm{g}$ of the anti-FLAG antibody M5 or an isotype control (C). After a 4 hour incubation, protein$\mathrm{G}$ sepharose was added and the mixtures were incubated for an additional 2 hours. Protein

complexes were washed three times with cold lysis buffer, eluted from beads by resuspending in 1× SDS-PAGE loading buffer, and Nedd4 westerns were performed as described above. Ponceau S staining of the blot after transfer revealed equivalent amounts of immunoprecipitating antibody. (B) Immunoprecipiations were performed as described in (A), except that the Nedd4 polyclonal was used in the immunoprecipitations and the M5 antibody was used for the western blot.

WW domain 1 mutant associates with Spt23, forming a complex that is resistant to Rsp5-induced processing. To determine if the WW domain 1 mutant interacts with Spt23, coimmunoprecipitation studies were carried out. Spt23 protein complexes were isolated by immunoprecipitation, separated by SDS-PAGE, and immunoblots were performed with a Nedd4 polyclonal antibody. In addition, we carried out the reciprocal approach where immunoprecipitations used the Nedd4 polyclonal and western blots employed an antibody that detects Spt23. As depicted in Fig. 3A, Nedd4 protein was detected in Spt23 immunoprecipitates derived from cells expressing the WW domain 1 mutant. Unprocessed but not processed Spt23 was detected in the Nedd4 immunoprecipitations (Fig. 3B), indicating that Nedd4 interacts preferentially with membranelocalized Spt23. We were able to detect an interaction between wt Nedd4 and membrane-bound Spt23 (Fig. 3B), although the amount of Spt23 in the complex with wt Nedd4 was much less than the amount present in complex with the WW domain 1 mutant. This could be due to the fact that either there is more membrane-localized Spt23 in cells expressing the WW domain 1 mutant or the wt Nedd4-Spt23 interaction is unstable. Nevertheless, these results indicate that the WW domain 1 mutant is not defective at interacting with Spt23.

It is known that the C-terminal WW domains of Nedd4 binds to proline-rich sequences found within its targets (Staub et al., 1996; Harvey et al., 1999; Abriel et al., 2000; Hamilton et al., 2001; Pham and Rotin, 2001). Interestingly, Spt23 does not contain any proline-rich sequences. To determine if Nedd4 binding to Spt23 requires its C-terminal localized WW domains, we tested WW domain 1 combination mutants for their ability to inhibit Spt23 processing and interact with Spt23. Fig. 4A shows that the WW domain $1 / 2,1 / 3$ and $1 / 2 / 3$ mutants do not alter Spt23 processing, indicating that WW domains 2 and 3 are both required for the dominant-negative activity of the WW domain 1 mutant. WW domain 4 is unlikely to be required for the activity of the WW domain 1 mutant as the WW domain 1/4 mutant induces accumulation of higher molecular weight Spt23 and inhibits generation of processed Spt23 (Fig. 4A). Coimmunoprecipitation studies were next performed to determine if mutations of WW domains 2 and/or 3 abrogate an interaction between the WW domain 1 mutant and Spt23 in cells. As shown in Fig. 4B, Nedd4 protein was present in Spt23 immunoprecipitates derived from cells expressing the WW domain 1 mutant but not in immunoprecipitates derived from cells producing the $\mathrm{WW} 1 / 2$, WW1/3 or WW1/2/3 mutants. Although these experiments do not give an indication whether the interaction between Nedd4 and Spt23 is direct, the results suggest that the formation of the Nedd4-Spt23 protein complex in cells require the cooperative binding function of WW domains 2 and 3 .

Rsp5 proteins harboring mutations in either WW domain 1 or the HECT domain block Spt23 processing

The results indicate that both WW domain 1 binding function and ubiquitin ligase activity is required for $\mathrm{Spt} 23$ processing by Nedd4 and Rsp5 in budding yeast. To determine whether this is the case, we tested Nedd4 and Rsp5 mutants lacking ligase function (harboring a C-terminal Cys mutation) and a Rsp5 WW domain 1 deletion mutant for their ability to alter Spt23 processing. Interestingly, similar to the catalytically dead Nedd4 and Rsp5 mutants and the WW domain 1 Nedd4 mutant, the Rsp5 mutant lacking WW domain 1 inhibits the production of processed Spt23 and induces accumulation of precursor Spt23 (Fig. 5A). As with the WW domain 1 Nedd4 mutant and Spt23, a protein complex comprising unprocessed Spt23 and the WW domain 1 Rsp5 mutant was easily detected by coimmunoprecipitation (Fig. 5B). These results indicate that similar to WW domain 1 of Nedd4, WW domain 1 of Rsp5 is dispensable for binding but not ubiquitin/proteasome-dependent processing of the membrane bound transcription factor Spt23.

\section{WW domain 1 and the C-terminal Cys are required for Nedd4-induced proteasome-mediated degradation of Spt23 in mammalian cells}

WW domain 1 is the most highly conserved of the WW domains between Rsp5 and Nedd4, and it likely that it performs a similar function in higher eukaryotes. Nedd4 has not yet been shown to induce ubiquitin/proteasome-dependent processing of any mammalian targets. Therefore, we used Spt23 as a model substrate to determine whether wt Nedd4 induces proteasome-dependent proteolysis of the protein in transfected mammalian cells and whether an this is dependent 
A.

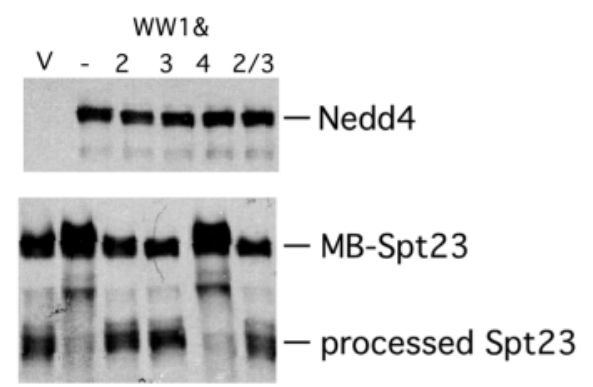

B.

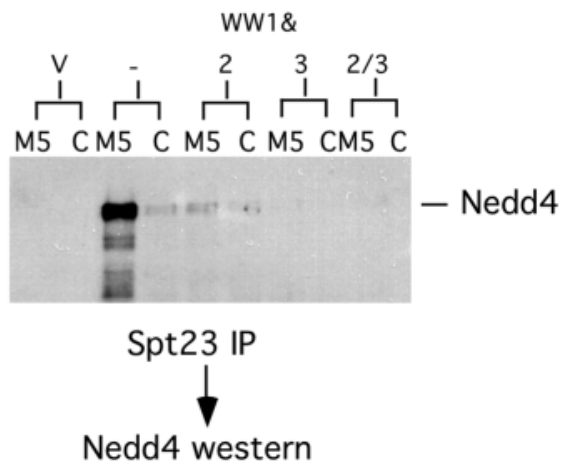

Fig. 4. Inhibition of Spt23 processing by the WW domain 1 mutants requires WW domains 2 and 3, and both of these C-terminal WW domains are required for Spt23 binding. (A) Cells were first transformed using the pESC-FLAG-Spt23 expression construct and then with $\mathrm{pYes}$ (v), pYes-wt Nedd4 (wt), pYes-WW1 (-) and pYes-WW domain 1 mutant Nedd4 expression vectors harboring substitutions in the indicated WW domains. Cells were processed as described in the legend of Fig. 2C. Measurement of Nedd4 protein in the extract was performed by a western blot using the anti-Nedd4 polyclonal while the amount of membrane-bound (MB) and processed Spt23 was measured by the anti-FLAG tag antibody M5. Ponceau S staining of the blot after transfer revealed equivalent loading of total protein. (B) An equal amount of extract prepared from cells producing FLAG-tagged Spt 23 and the indicated Nedd4 proteins were subjected to immunoprecipitation with the anti-FLAG antibody M5 or an isotype control antibody (C). The amount of Nedd4 protein immunoprecipitated with Spt 23 was then determined by a western blot. Ponceau S staining of the blot after transfer revealed equivalent amounts of immunoprecipitating antibody.

on WW domain 1 or the C-terminal Cys residue. Human H1299 cells were transfected with a mammalian Spt23 expression vector alone or with expression constructs encoding various Nedd4 proteins (performed in duplicate). One set of transfections was left untreated while the other was treated with the proteasome inhibitor MG115. Cells were harvested and an immunoblot was performed to assess Spt23 expression status. It appears that the mechanism regulating Spt23 processing is conserved since the expression pattern of Spt23 in transfected mammalian cells is similar to that observed in yeast (Fig. 6). Moreover, treatment of cells with MG115 leads to an increase in unprocessed Spt23 and reduces the amount of lower molecular weight product. Interestingly, wt Nedd4 induces degradation of unprocessed Spt23 and this is blocked by MG115 (Fig. 6). Fig. 6 also demonstrates that Nedd4 mutants harboring substitutions in WW domain 1 or the ligase domain are unable to induce $\mathrm{Spt} 23$ degradation. In addition, the level of unprocessed Spt23 precursor is higher in cells transfected with these mutants, indicating that they interact with Spt23 and prevent degradation by endogenous proteins. We conclude from these experiments that the WW domain 1 of Nedd4 interacts with a protein that promotes ubiquitin/ proteasome-dependent degradation of $\mathrm{Spt} 23$ in mammalian cells.

\section{Discussion}

E3s, or ubiquitin ligases, represent the specificity arm of the ubiquitin-proteolytic pathway (Hershko and Ciechanover, 1998; Pickart, 2001). Mammalian Nedd4 and budding yeast Rsp5 are members of a large family of E3 that contain a HECT domain, a C2 domain and multiple WW domains (Harvey and Kumar, 1999). The HECT domain promotes the transfer of ubiquitin from an E2 enzyme to a bound target whereas the C2 domain appears to control membrane localization of the proteins. The C-terminal WW domains interact with targets but the function of WW domain 1 remains undefined.
We have found that ectopic expression of Nedd4 or Rsp5 mutants, which have alterations in WW domains, blocks processing of the membrane-bound transcription factor Spt23 in budding yeast. Considering that the WW domain 1 mutants are present in a Spt 23 protein complex, it is likely that they are able to interact with Spt23 (either directly or through adapter proteins) but are unable to promote proteasome-dependent processing. Therefore, it is reasonable to speculate on the basis of this data that the WW domain 1 binds to a cofactor in yeast cells that is required for ubiquitin/proteasome-dependent processing of Spt23 by Rsp5 and Nedd4. The cofactor may promote the attachment of ubiquitin to a specific site on Spt23 that is needed for processing, control ubiquitin chain assembly on Spt23, which confers a specific type of degradation signal or modulate the folding of Spt23 so that it is processed in a limited manner. Past studies have shown that Bul1 and Bul2 function in similar pathways to Rsp5 (Fisk et al., 1999; Wolfe et al., 1999; Helliwell et al., 2001; Soetens et al., 2001). Interestingly, Bul1 interacts with Rsp5 but is not a substrate of the ligase (Yashiroda et al., 1996; Yashiroda et al., 1998). Also, it has been demonstrated that Bul1 and Bul2 are involved in Rsp5-induced degradation of Rog1 (Andoh et al., 2000), raising the possibility that they work either as adapter proteins or as cofactors in the degradation of a subset of Rsp5 targets. We are currently testing whether Bul1 and Bul2 bind to WW domain 1 of Rsp5 and promote Spt23 processing. Obviously, future studies are warranted to identify WW-domain1-binding factors and determine their mechanisms of action.

Although mutation in WW domain 1 of Rsp5 affects fluidphase endocytosis (Dunn and Hicke, 2001; Gajewska et al., 2001), WW domains 1 and 2 of Rsp5 are dispensable for its essential function under normal growth conditions (Hoppe et al., 2000; Gajewska et al., 2001). Therefore, it is possible that WW domain 1 is not required for Rsp5-induced processing of MGA2, the other membrane-bound transcription factor that performs redundant functions with Spt23 and is involved in ole1 transactivation (Zhang et al., 1999). Alternatively, the 
Fig. 5. Spt23 processing is inhibited by WWdomain 1- or ligase-defective Nedd4 and Rsp5 mutants. (A) Cells were transformed first with the pESC-FLAG-Spt23 expression construct and then with the indicated Nedd4 and Rsp5 expression constructs. Cells were processed as described in the legend of Fig. 2C. The amount of membranebound (MB) and processed Spt 23 was measured by the anti-FLAG tag antibody M5. Ponceau S staining of the blot after transfer revealed equivalent loading of total protein. (B) Equal amount of extract prepared from cells producing FLAG-tagged Spt23 and the indicated Rsp5 proteins were subjected to immunoprecipitation with the anti-HA antibody 12CA5 (12) or an isotype control antibody (C). The amount of Nedd4 protein immunoprecipitated with Spt23 was then determined by a western blot. Ponceau S staining of the blot after transfer revealed equivalent amounts of immunoprecipitating antibody.

WW-domain1-binding protein may interact with multiple Rsp5 WW domains. Considering that Rsp5 interacts with itself (Dunn and Hicke, 2001), it is plausible that under conditions where WW domains 1 and 2 are not operational, binding to the target is mediated via WW domain 3 of one molecule of the Rsp5 dimer while binding to the cofactor occurs through the other. Nevertheless, it will be interesting to determine if the WW domain 1 Rsp5 mutant possesses dominant negative activity on other Rsp5 targets, including MGA2 and those that function in different endocytosis pathways (Wang et al., 1999; Dunn et al., 2001; Gajewska et al., 2001).

WW domain 1 of Nedd4 probably performs cofactor functions in mammalian cells. Utilizing Spt23 as a model substrate, we found that wt Nedd4 induces proteasomedependent degradation of unprocessed Spt23, and this activity is dependent on WW domain 1 and the C-terminal Cys. Moreover, because these mutants induce stabilization of unprocessed Spt23, it is highly probable that they form protein complexes with full-length $\mathrm{Spt} 23$ in transfected mammalian cells. The abundance of ectopically expressed proteins in transfected H1299 cells is not nearly as high as in yeast, making it difficult to detect an association by

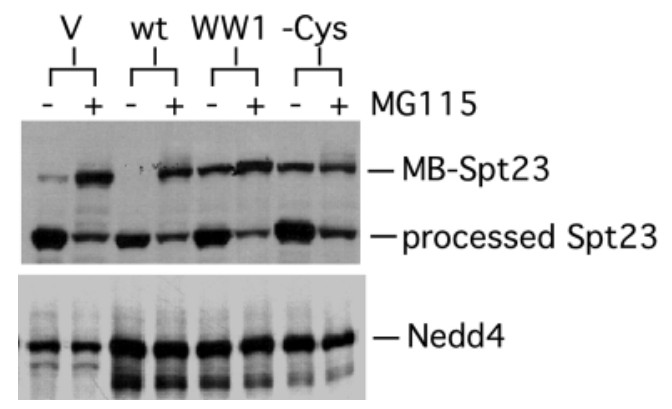

Fig. 6. The WW domain 1 is required for proteasome-dependent degradation of Spt23 in mammalian cells. H1299 cells were transfected with pCEP-FLAG-Spt23 and pCEP (V), pCEP-wt Nedd4 (wt) or pCEP containing the indicated Nedd4 mutants by Lipofectamine. After transfection, cells were re-fed with complete media (-) or media containing the proteasome inhibitor MG115 (+). Cells were incubated for 9 hours prior to harvesting. Extracts were prepared as described in the Materials and Methods and western blots were performed with the Nedd4 polyclonal or the anti-FLAG antibody M5. Ponceau S staining of the blot after transfer revealed equivalent loading of total protein. coimmunoprecipitation. Interestingly, wt Nedd4 does not promote degradation of precursor Spt 23 in yeast. Although we can not rule out the possibility that Nedd4 induces partial proteolysis that leads to total degradation of precursor, the results raise the possibility that the WW-domain1-binding factor in H1299 cells promotes complete rather than partial destruction of this substrate. Processed Spt23 is probably being generated by another E3 ligase or a cotranslational proteasomedependent processing mechanism (Lin et al., 1998) that is not susceptible to the dominant-negative activity of the WW domain 1 or C-terminal Cys mutants. The WW domain 1 has been reported to be required for downregulation of epithelial $\mathrm{Na}^{+}$channel activity even though it does not interact with channel subunits (Harvey et al., 1999). It has been postulated that the WW domain 1 interacts with a protein that recruits Nedd4 to the channel or stabilizes the interaction between Nedd4 and the epithelial $\mathrm{Na}^{+}$channel subunits (Harvey et al., 1999). On the basis of our work with Spt23, we speculate that Nedd4 WW-domain1-binding proteins function as cofactors in Nedd4-induced ubiquitination and degradation of substrates.

We thank L. W. Bergman for advice relating to yeast and E. BullGauntner for providing technical help in the early stages of the study. Also, we thank D. Rotin, O. Staub, J. Huibregtse and R. HaguenauerTsapis for helpful discussions and R. Haguenauer-Tsapis for her critical review of the manuscript. We acknowledge support from Public Health Service grant CA70165 and the Fels Foundation (to DSH).

\section{References}

Abriel, H., Kamynina, E., Horisberger, J. D. and Staub, O. (2000). Regulation of the cardiac voltage-gated Na+ channel (H1) by the ubiquitinprotein ligase Nedd4. FEBS Lett. 466, 377-380.

Andoh, T., Hirata, Y. and Kikuchi, A. (2000). Yeast glycogen synthase kinase 3 is involved in protein degradation in cooperation with Bul1, Bul2, and Rsp5. Mol. Cell. Biol. 18, 6712-6720.

Boyd, M. T., Vlatkovic, N. and Haines, D. S. (2000). A novel cellular protein (MTBP) binds to MDM2 and induces a G1 arrest that is suppressed by MDM2. J. Biol. Chem. 275, 31883-31890.

Chang, A., Cheng, S., Espanel, X. and Sudol, M. (2000). Rsp5 WW domains interact directly with the carboxyl-terminal domain of RNA polymerase II. J. Biol. Chem. 275, 20556-20571.

Dunn, R. and Hicke, L. (2001). Domains of the Rsp5 ubiquitin-protein ligase required for receptor-mediated and fluid-phase endocytosis. Mol. Biol. Cell 12, 421-435.

Erdeniz, N. and Rothstein, R. (2000). Rsp5, a ubiquitin-protein ligase, is involved in degradation of the single-stranded-DNA binding protein rfa1 in Saccharomyces cerevisiae. Mol. Cell. Biol. 20, 224-232.

Farr, T. J., Coddington-Lawson, S. J., Snyder, P. M. and McDonald, F. J. 
(2000). Human Nedd4 interacts with the human epithelial Na+ channel: WW3 but not WW1 binds to Na+ channel subunits. Biochem. J. 345, 503509.

Fisk, H. A. and Yaffe, M. P. (1999). A role for ubiquitination in mitochondrial inheritance in Saccharomyces cerevisiae. J. Cell Biol. 145, 1199-1208.

Gajewska, B., Kaminska, J., Jesionowska, A., Martin, N. C., Hopper, A. K. and Zoladek, T. (2001). WW domains of Rsp5p define different functions: determination of roles in fluid phase and uracil permease endocytosis in Saccharomyces cerevisiae. Genetics 157, 91-101.

Guthrie, C. and Fink, G. R. (1991). Guide to Yeast Genetics and Molecular Biology, Academic Press, London.

Hamilton, M. H., Tcherepanova, I., Huibregtse, J. M. and McDonnell, D. P. (2001). Nuclear import/export of hRPF1/Nedd4 regulates the ubiquitindependent degradation of its nuclear substrates. J. Biol. Chem. 276, 2632426331

Harvey, K. F. and Kumar, S. (1999). Nedd4-like proteins: an emerging family of ubiquitin protein ligases implicated in diverse cellular functions. Trends Cell Biol. 9, 166-169.

Harvey, K. F., Dinudom, A., Komwatana, P., Jolliffe, C. N., Day, M. L., Parasivam, G., Cook, D. I. and Kumar, S. (1999). All three WW domains of murine Nedd4 are involved in the regulation of epithelial sodium channels by intracellular Na+. J. Biol. Chem. 274, 12525-12530.

Helliwell, S. B., Losko, S. and Kaiser, C. A. (2001). Components of a ubiquitin ligase complex specify polyubiquitination and intracellular trafficking of the general amino acid permease. J. Cell Biol. 153, 649-662.

Hershko, A. and Ciechanover, A. (1998). The ubiquitin system. Annu. Rev. Biochem. 67, 425-479.

Hicke, L. (1999). Gettin' down with ubiquitin: turning off cell-surface receptors, transporters and channels. Trends Cell Biol. 9, 107-112.

Hitchcock, A. L., Krebber, H., Frietze, S., Lin, A., Latterich, M. and Silver, P. A. (2001). The conserved npl4 protein complex mediates proteasomedependent membrane-bound transcription factor activation. Mol. Biol. Cell 12, 3226-3241.

Hoppe, T., Matuschewski, K., Rappe, M., Schlenker, S., Ulrich, H. D. and Jentsch, S. (2000). Activation of a membrane-bound transcription factor by regulated ubiquitin/proteasome dependent processing. Cell 102, 577586.

Huibregtse, J. M., Scheffner, M., Beaudenon, S. and Howley, P. M. (1995). A family of proteins structurally and functionally related to the E6-AP ubiquitin-protein ligase. Proc. Natl. Acad. Sci. USA 92, 2563-2567.

Huibregtse, J. M., Yang, J. C. and Beaudenon, S. L. (1997). The large subunit of RNA polymerase II is a substrate of the Rsp5 ubiquitin-protein ligase. Proc. Natl. Acad. Sci. USA 94, 3656-3661.

Kaiser, P., Flick, K., Wittenberg, C. and Reed, S. I. (2000). Regulation of transcription by ubiquitination without proteolysis: Cdc34/SCF(Met30)mediated inactivation of the transcription factor Met4. Cell 102, 303-314.

Lin, L., DeMartino, G. N. and Greene, W. C. (1998). Cotranslational biogenesis of NF-kappaB p50 by the 26S proteasome. Cell 92, 819-828.

Lu, P. J., Zhou, X. Z., Shen, M. and Lu, K. P. (1999). Function of WW domains as phosphoserine- or phosphothreonine-binding modules. Science 283, 1325-1328.

Palombella, V. J., Rando, O. J., Goldberg, A. L. and Maniatis, T. (1994).
The ubiquitin-proteasome pathway is required for processing the NF-kappa B1 precursor protein and the activation of NF-kappa B. Cell 78, 773-785

Pham, N. and Rotin, D. (2001). Nedd4 regulates ubiquitination and stability of the guanine nucleotide exchange factor CNrasGEF. J. Biol. Chem. [epub ahead of print].

Pickart, C. M. (2001) Mechanisms underlying ubiquitination. Annu. Rev. Biochem. 70, 503-533.

Plant, P. J., Yeger, H., Staub, O., Howard, P. and Rotin, D. (1997). The C2 domain of the ubiquitin protein ligase Nedd4 mediates $\mathrm{Ca} 2+$-dependent plasma membrane localization. J. Biol. Chem. 272, 32329-32336.

Plant, P.J., Lafont, F., Lecat, S., Verkade, P., Simons, K. and Rotin, D. (2000). Apical membrane targeting of Nedd4 is mediated by an association of its C2 domain with annexin XIIIb. J. Cell Biol. 149, 1473-1484.

Rizo, J. and Südhof, T. C. (1998). C2-domains, structure and function of a universal Ca2+ binding domain. J. Biol. Chem. 273, 15879-15882.

Rotin, D., Staub, O. and Haguenauer-Tsapis, R. (2000). Ubiquitination and endocytosis of plasma membrane proteins: role of Nedd4/Rsp5p family of ubiquitin-protein ligases. J. Membr. Biol. 176, 1-17.

Soetens, O., De Craene, J. O. and Andre, B. (2001). Ubiquitin is required for sorting to the vacuole of the yeast Gap1 permease. J. Biol Chem. Aug 10 [epub ahead of print].

Staub, O., Dho, S., Henry, P., Correa, J., Ishikawa, T., McGlade, J. and Rotin, D. (1996). WW domains of Nedd4 bind to the proline-rich PY motifs in the epithelial $\mathrm{Na}+$ channel deleted in Liddle's syndrome. EMBO J. 15, 2371-2380.

Stukey, J. E., McDonough, V. M. and Martin, C. E. (1989). Isolation and characterization of OLE1, a gene affecting fatty acid desaturation from Saccharomyces cerevisiae. J. Biol. Chem. 264, 16537-16544.

Sudol, M. and Hunter, T. (2000). NeW wrinkles for an old domain. Cell 103, 1001-1004.

Wang, G., Yang, J. and Huibregtse, J. M. (1999). Functional domains of the Rsp5 ubiquitin protein ligase. Mol. Cell. Biol. 19, 342-352.

Wang, G., McCaffery, J. M., Wendland, B., Dupre, S., Haguenauer-Tsapis, R. and Huibregtse, J. M. (2001). Localization of the Rsp5p ubiquitinprotein ligase at multiple sites within the endocytic pathway. Mol. Cell. Biol. 21, 3564-3575.

Wang, C., Deng, L., Hong, M., Akkaraju, G. R., Inoue, J. and Chen, Z. J. (2001). TAK 1 is a ubiquitin-dependent kinase of MKK and IKK. Nature 412, 346-351.

Wolfe, D., Reiner, T., Keeley, J. L., Pizzini, M. and Keil, R. L. (1999). Ubiquitin metabolism affects cellular response to volatile anesthetics in yeast. Mol. Cell. Biol. 19, 8254-8262.

Yashiroda, H., Oguchi, T., Yasuda, Y., Toh-E., A. and Kikuchi, Y. (1996). Bul1, a new protein that binds to the Rsp5 ubiquitin ligase in Saccharomyces cerevisiae. Mol. Cell. Biol. 16, 3255-3263.

Yashiroda, H., Kaida, D., Toh-E., A. and Kikuchi, Y. (1998). The PY-motif of Bul1 protein is essential for growth of Saccharomyces cerevisiae under various stress conditions. Gene 225, 39-46.

Zhang, S., Skalsky, Y. and Garfinkel, D. J. (1999). MGA2 or SPT23 is required for transcription of the delta9 fatty acid desaturase gene, OLE1, and nuclear membrane integrity in Saccharomyces cerevisiae. Genetics $\mathbf{1 5 1}$, 473-483. 